\title{
Ultrastructure of the anterior organ and posterior funnel-shaped canal of Gyrocotyle urna Wagener, 1852 (Cestoda: Gyrocotylidea)
}

\author{
Larisa G. Poddubnaya ${ }^{1}$, Roman Kuchta ${ }^{2}$, Glenn A. Bristow ${ }^{3,4}$ and Tomáš Scholz ${ }^{2}$ \\ ${ }^{1}$ I.D. Papanin Institute for Biology of Inland Waters, Russian Academy of Sciences, Borok, Yaroslavl Province, Russia; \\ ${ }^{2}$ Institute of Parasitology, Biology Centre of the Czech Academy of Sciences ,České Budějovice, Czech Republic; \\ ${ }^{3}$ Department of Biology, University of Bergen, Bergen, Norway; \\ ${ }^{4}$ Institute of Biotechnology and Environment, Nha Trang University, Nha Trang, Vietnam
}

\begin{abstract}
Using scanning and transmission electron microscopy, ultrastructure of the anterior organ and posterior funnel-shaped canal of Gyrocotyle urna Wagener, 1852 (Cestoda: Gyrocotylidea) from ratfish, Chimaera monstrosa (Holocephali), was studied for the first time. The proper anterior organ is localised at a short distance (about $170 \mu \mathrm{m}$ ) from an apical pore surrounded by a receptor field, whereas its distal end is marked by a muscular sphincter. The tegumental surface of this organ is covered with short filitriches of irregular length; large area of muscle layers traverse beneath the tegumental layer. The funnel-shaped canal of G. urna (2.5-3.0 mm long) is a specialised, muscular part of the posterior attachment organ; it opens on the rounded elevation on the dorsal body surface. The tegumental layer bears conical sclerite-like structures (up to $1.5 \mu \mathrm{m}$ long). It produces electron-dense bodies that are transported into a canal lumen and surrounded thick muscle area mixed with numerous nerve fibres. The present ultrastructural study of $G$. urna indicates that gyrocotylideans share some ultrastructural characters of the anterior organ with spathebothriidean cestodes with a single anterior attachment sucker-like organ. In contrast, the unique posterior rosette attachment organ with funnel-shaped canal of the Gyrocotylidea resembles the haptor of polyopisthocotylean monogeneans in its position at the posterior end of the body and presumed origin. The above-mentioned features add more clarity to support the basal position of the Gyrocotylidea Poche, 1926 among cestodes. In addition, they also indicate a possible relationship of gyrocotylidean ancestors with monogeneans.
\end{abstract}

Keywords: scanning electron microscopy, transmission electron microscopy, basal cestodes, ultrastructural characters, phylogeny

The Gyrocotylidea Poche, 1926 are a small and enigmatic group of unsegmented cestodes (Cestoda), which includes 10 nominal species that are parasites of ratfishes (also known as chimaeras) belonging to an ancient vertebrate group, the Holocephali (Bandoni and Brooks 1987, Williams et al. 1987, Bristow 1992, Gibson 1994). The phylogenetic relationships of gyrocotylideans to other neodermatans, i.e. derived parasitic flatworms with a neodermis, have been a matter of discussion for a long time (Bandoni and Brooks 1987, Olson et al. 2001, Xylander 2001, Lockyer et al. 2003, Waeschenbach et al. 2012).

The absence of a digestive system places gyrocotylideans in the class Cestoda, even though the morphology of gyrocotylideans is unique among cestodes, because they possess paired anterior excretory pores, a peculiar arrangement of genital pores, a male penis, a follicular ovary located anteriorly, and a posterior haptor-like organ (rosette). In the possession of these characteristics, gyrocotylideans somewhat resemble monogeneans. However, molecular data have unequivocally confirmed that the Gyrocotylidea is the most basal order of the Cestoda (Waeschenbach et al. 2007, 2012).

The Gyrocotylidea are also characterised by the presence of an anterior organ that appears as an invagination of the anterior extremity of the body. Brooks at al. (1985) compared this invagination with the mouth (oral sucker) of monogeneans, testifying to the ancestral presence of a digestive system and considering this structure as muscular organ; they illustrated this organ as a sucker for 10 species of Gyrocotyle Wagener, 1852 (see Bandoni and Brooks 1987). However, no detailed ultrastructural study has been carried out to provide reliable information on the internal structure of this peculiar organ.

The posterior rosette of gyrocotylideans serves for their fixation in the spiral valve of the final (definitive) hosts (Williams et al. 1987), a conspicuous difference from other cestodes where the attachment organs are situated at the anterior extremity of the body (scolex). The posterior at- 
tachment rosette organ of the Gyrocotylidea represents the folded border of a funnel-shaped canal extending from the posterior end of the body to its dorsal pore not far from the rosette (Watson 1911, Halvorsen and Williams 1968, Williams et al. 1987).

The aim of the present study is to provide data on the ultrastructure of the anterior organ at the anterior end and the funnel-shaped canal of the posterior attachment organ of Gyrocotyle urna Wagener, 1852. This account is a follow-up of our previous ultrastructural studies on these enigmatic flatworms (Poddubnaya et al. 2006, 2008, 2009, 2010, Xylander and Poddubnaya 2009), helping to obtain additional ultrastructural characters suitable for the reconstruction of the evolutionary history of basal tapeworms and their functional morphology.

\section{MATERIALS AND METHODS}

Mature and immature specimens of Gyrocotyle urna were removed from the spiral valve of chimaera, Chimaera monstrosa Linnaeus (Holocephali), from deep-water, within the 500-750 m bathymetric zones of the North Sea off Bergen, Norway in May 2010. The fish were caught aboard the RV 'Hans Brattström' belonging to the University of Bergen using a deep-water trawl.

For electron microscopy, entire worms were fixed using 3\% glutaraldehyde in $0.1 \mathrm{M}$ sodium cacodylate buffer $(\mathrm{pH}$ 7.2) for 20 days at $5^{\circ} \mathrm{C}$, rinsed 4 times for $20 \mathrm{~min}$ in the same buffer and post-fixed in $1 \%$ osmium tetroxide for $1 \mathrm{~h}$.

For transmission electron microscopical (TEM) examinations, samples were then dehydrated in a graded series of ethanol and acetone, and embedded in a mixture of Araldite and Epon. U1trathin sections (70-90 $\mathrm{nm}$ in thickness) were stained with uranyl acetate and lead citrate, and examined using a JEOL-JEM-1011 transmission electron microscope operating at $80 \mathrm{kV}$.

For scanning electron microscopical (SEM) observations, fixed worms were dehydrated in a graded ethanol series, with a final change in absolute ethanol, and then critical-point-dried with liquid $\mathrm{CO}_{2}$. The specimens were mounted on stubs, sputter-coated with gold-palladium and examined using JEOL-JSM-6510LV scanning electron microscope operating at $15 \mathrm{kV}$.

For histological study, specimens were fixed in hot $4 \%$ formaldehyde solution, dehydrated through an ethanol series and embedded in paraffin. Sections (thickness $12 \mu \mathrm{m}$ ) were stained with haematoxylin and eosin, mounted in Canada balsam and examined using an Olympus BX-51 microscope. Terminology for cestode microtriches follows Chervy 2009.

\section{RESULTS}

\section{Anterior organ of Gyrocotyle urna}

SEM micrographs of Gyrocotyle urna show that its anterior extremity can be slightly extended over the body and is pierced by a circular opening (about $270 \mu \mathrm{m}$ in diameter) into the cavity of the anterior organ (Fig. 1A,C). It represents a closed invagination of the anterior extremity of the body tegument, surrounded by a thick muscle area (Fig. 1B). The proper anterior organ is localised at a short distance (about $170 \mu \mathrm{m}$ ) from the apical pore, with its distal end marked by a muscular sphincter that can be observed on both sides of the apical invagination in histological and ultrathin sections (Fig. 1B,G). The proper anterior organ is about $2.5 \mathrm{~mm}$ long and up to $1.5 \mathrm{~mm}$ wide, slightly compressed dorsoventrally. The area of the muscular sphincter is oval in shape (about $150 \mu \mathrm{m}$ long and $90 \mu \mathrm{m}$ wide) and contains closely-packed longitudinal muscle fibrils, with mitochondria at the periphery of their sarcoplasm (Fig. 1F).

The lumen of the anterior organ is lined by a syncytial tegument that is a prolongation of that of the main body (Fig. 1B,G). From the apical pore and distal portion, up to the muscular sphincter, the syncytial tegumental lining is about $21 \mu \mathrm{m}$ thick and thickness of the lining of the proper anterior organ is about $9 \mu \mathrm{m}$. The tegumental surface around the apical pore is covered with capilliform filitriches (Fig. 1E), which are progressively shorter from the distal to proximal portions of the anterior invagination. The capilliform filitriches of the tegument around and on the anterior organ have short electron-dense tips (Fig. 1H). Mitochondria, vesicles and dense bodies are present in the syncytial cytoplasm of the body tegument around the pore of the anterior organ (Fig. 1H). The syncytial cytoplasm of the proper anterior organ contains numerous vacuoles scattered throughout and arranged in a regular row just above the basal membrane, and many dense bodies (Fig. 2F). The tegument is based on a distinct basal lamina (Fig. 2F).

At the pore, the tegument bears different types of receptors that are grouped together in groups of 2-6 receptors of both uniciliate and aciliate types (Fig. 1D,E,H), but they are not examined in detail in the present paper. The uniciliate type of receptor is situated within the tegument close to the surface; its cilium is situated between surface folds or can project beyond filitriches of the tegument (Fig. 1E). Their bulbous ending is characterised by the presence of one or two peripherally situated electron-dense collars (Fig. 1H). Receptors of the aciliate type are localised in the proximal region of the tegument and characterised by two dense collars within the dendrite (Fig. 1H).

The anterior organ is a muscular one (Figs. 1B, 2A). Muscle layers traverse the region of this organ in various directions (Fig. 2B,E). Densely-packed muscle fibrils extend from the body proper into the stalk and anterior organ, crossing each other. In the anterior organ itself, they extend beneath and along the tegument and obliquely in several directions (Fig. 2B,E). Numerous cytons are present between muscle layers (Fig. 2E,G). The musculature around and inside the anterior organ is supplied with numerous nerve fibres that enter the muscle layers through the anterior nerve cord with the anterior nerve commissure lying around and just in front of the posterior margin of the anterior organ; its muscular sphincter is close to the anterior nerve cord (Fig. 2B-E). The nerve cord consists of a neuropile and a series of neurosecretory neurons distributed along the nerve cord (Fig. 2C). The neuropile contains a large number of nerve fibres, filled with diverse neurovesicles; the fibres are interconnected by synapses (Fig. 2D).

\section{Posterior funnel-shaped canal of Gyrocotyle urna}

The posterior margin of the funnel-shaped canal of G. urna is bordered by a large number of closely crumpled folds, which make up the posterior rosette (Fig. 3A). 

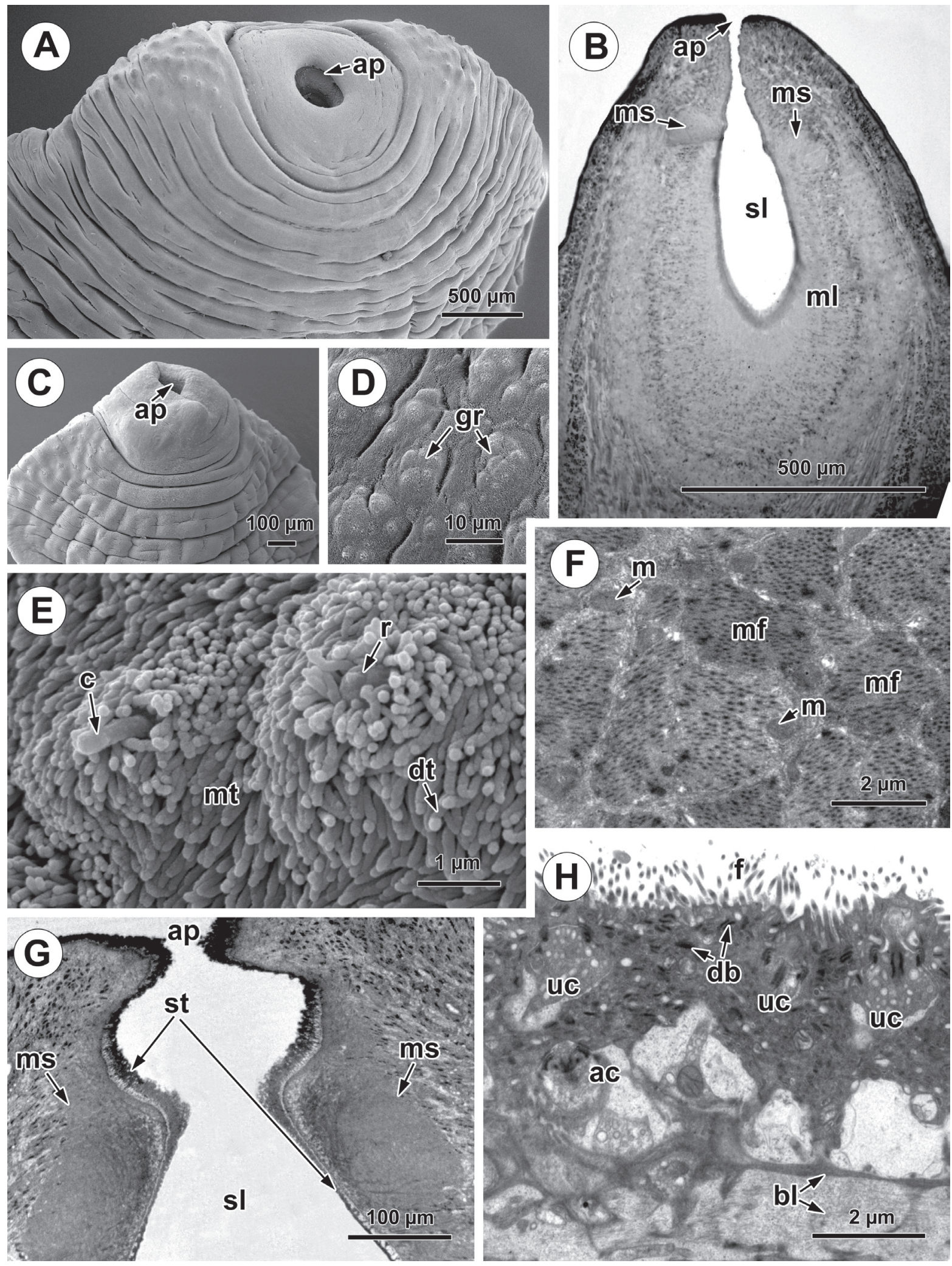

Fig. 1. Anterior organ of mature Gyrocotyle urna Wagener, 1855 from Chimaera monstrosa Linnaeus. A - Scanning electron micrographs (SEM) of the anterior part of the body; $\mathbf{B}$ - histological section of the anterior organ of adult worm showing the closed invagination of the anterior extremity of the body surrounded by muscle layers; $\mathbf{C}-\mathrm{SEM}$ view of the slightly extruded anterior extremity of the body; D - SEM view of the surface around the apical pore showing groups of receptors; $\mathbf{E}$ - SEM micrograph of the surface around the pore showing capilliform filitriches and two receptors; $\mathbf{F}$ - Transmission electron micrograph of closely packed muscle fibrils of the muscular sphincter; $\mathbf{G}$ - TEM view of the distal portion of the anterior invagination; $\mathbf{H}$ - group of receptors on the surface of the distal portion of the anterior invagination. Abbreviations: ac - aciliate receptor; ap - apical pore of the anterior organ; bl - basal lamina of tegumental syncytial cytoplasm; $\mathrm{c}$ - cilium of the receptor; $\mathrm{db}$ - dense bodies; $\mathrm{dt}$ - dense tip of filitriches; $\mathrm{f}$ - filitriches; gr - group of receptors; $\mathrm{m}$ - mitochondrion; $\mathrm{mf}$ - muscle fibrils; $\mathrm{ml}$ - muscle layers; $\mathrm{ms}$ - muscular sphincter; $\mathrm{mt}$ - filiform microtriches (filitriches); $\mathrm{r}$ - receptor; sl - lumen of the anterior organ; st - syncytial tegument of the anterior organ; uc - uniciliate receptor. 

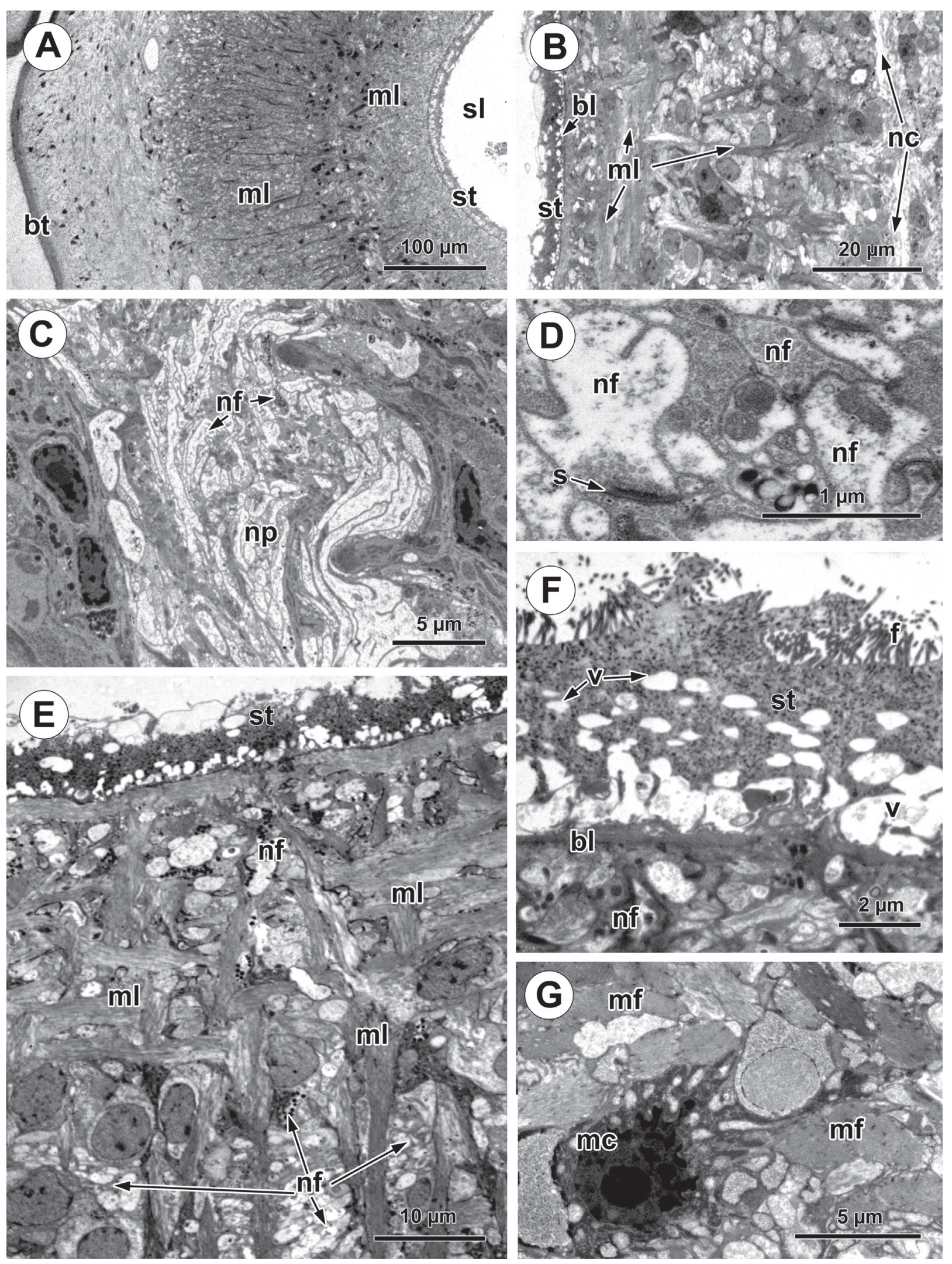

Fig. 2. Ultrastructure of the anterior organ of immature and mature Gyrocotyle urna Wagener, 1855 from Chimaera monstrosa Linnaeus; transmission electron micrographs. A - Portion of the anterior organ, showing numerous muscle layers surrounding its syncytial tegumental cytoplasm; $\mathbf{B}$ - portion of the anterior organ of immature worm; $\mathbf{C}$ - portion of the nerve cord, neuropile of which contains numerous nerve fibres; $\mathbf{D}$ - high magnification of nerve fibres, two of them interconnected by synapsis; $\mathbf{E}$ - portion of the anterior organ of an immature worm; $\mathbf{F}$ - tegumental syncytial cytoplasm of the anterior organ; $\mathbf{G}$ - muscle cell and numerous muscle fibrils of the anterior organ musculature. Abbreviations: bl - basal lamina of tegumental syncytial cytoplasm; bt - body tegument; mc - muscle cell; $\mathrm{mf}$ - muscle fibrils; $\mathrm{ml}$ - muscle layers; $\mathrm{nc}$ - nerve cord; $\mathrm{nf}$ - nerve fibres; $\mathrm{np}$ - neuropile; $\mathrm{s}$ - synapse; $\mathrm{sl}$ - lumen of the anterior organ; st - syncytial tegument of the anterior organ; v-vacuole. 

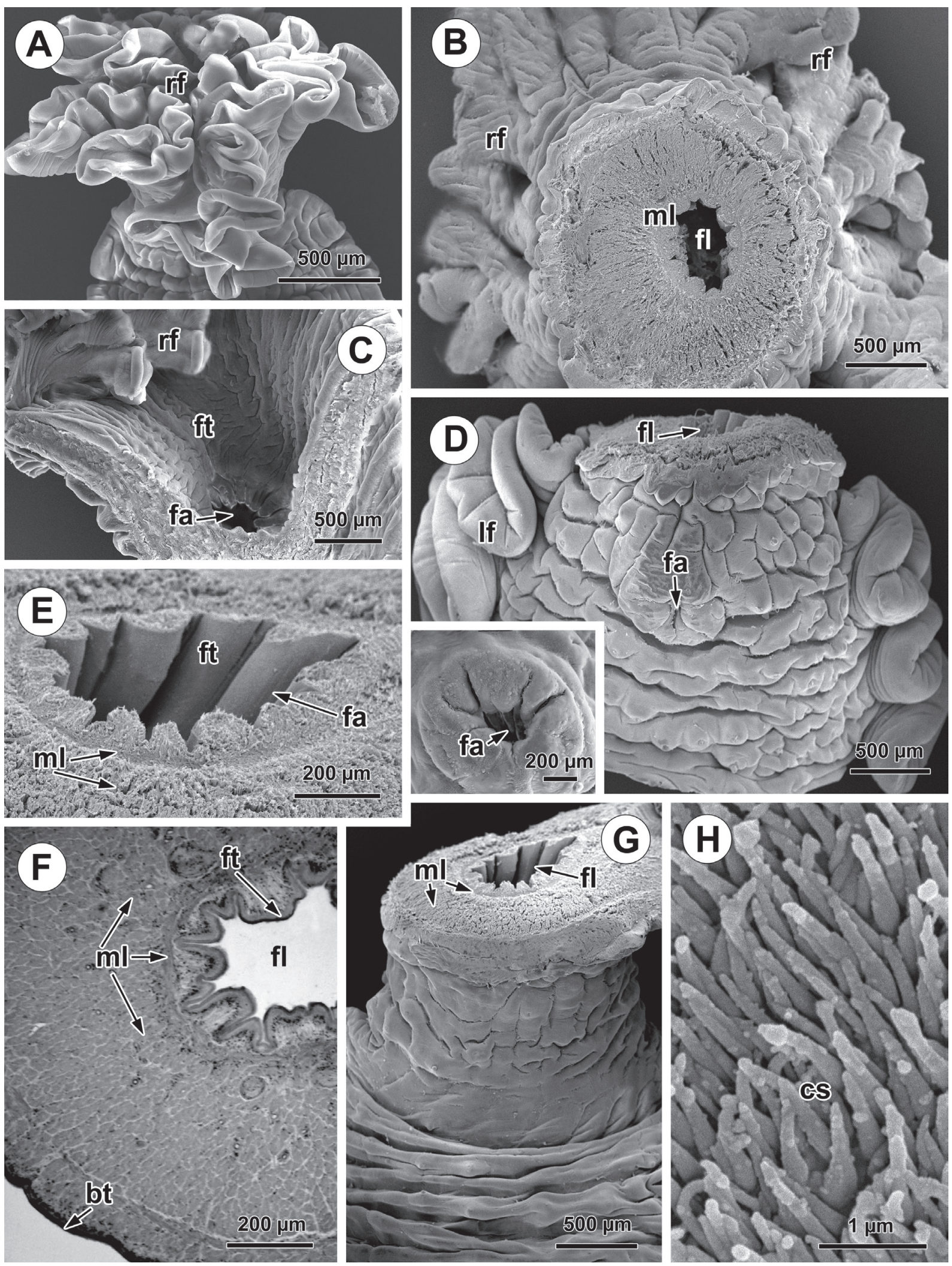

Fig. 3. Funnel-shaped canal of mature Gyrocotyle urna Wagener, 1855 from Chimaera monstrosa Linnaeus (A-E,G,H - scanning electron micrographs). A - folds of the posterior rosette attachment organ; $\mathbf{B}$ - cross section of the funnel-shaped canal cut near the posterior body end; $\mathbf{C}$ - longitudinal funnel-shaped canal sectioned through the whole canal, showing its luminal surface, rosette folds and the aperture of the funnel-shaped canal; $\mathbf{D}$ - posterior end portion; note aperture of the funnel-shaped canal on rounded elevation; inset - view of the rounded elevation with aperture; $\mathbf{E}$ - cross section through the funnel-shaped canal; $\mathbf{F}$ - histological section of the funnel-shaped canal; note the tegument of the canal lumen surrounding muscle layers and body tegument; $\mathbf{G}-$ view of the posterior end with cross section through the funnel-shaped canal; $\mathbf{H}$ - tegument of the funnel-shaped canal covered with conical surface structures. Abbreviations: bt - body tegument; cs - conical surface structures of the funnel-shaped canal tegument; fa - funnel-shaped canal aperture; $\mathrm{fl}$ - funnel-shaped canal lumen; $\mathrm{ft}$ - funnel-shaped canal tegument; lf - lateral body fold; ml - muscle layers; $\mathrm{rf}$ - rosette folds. 

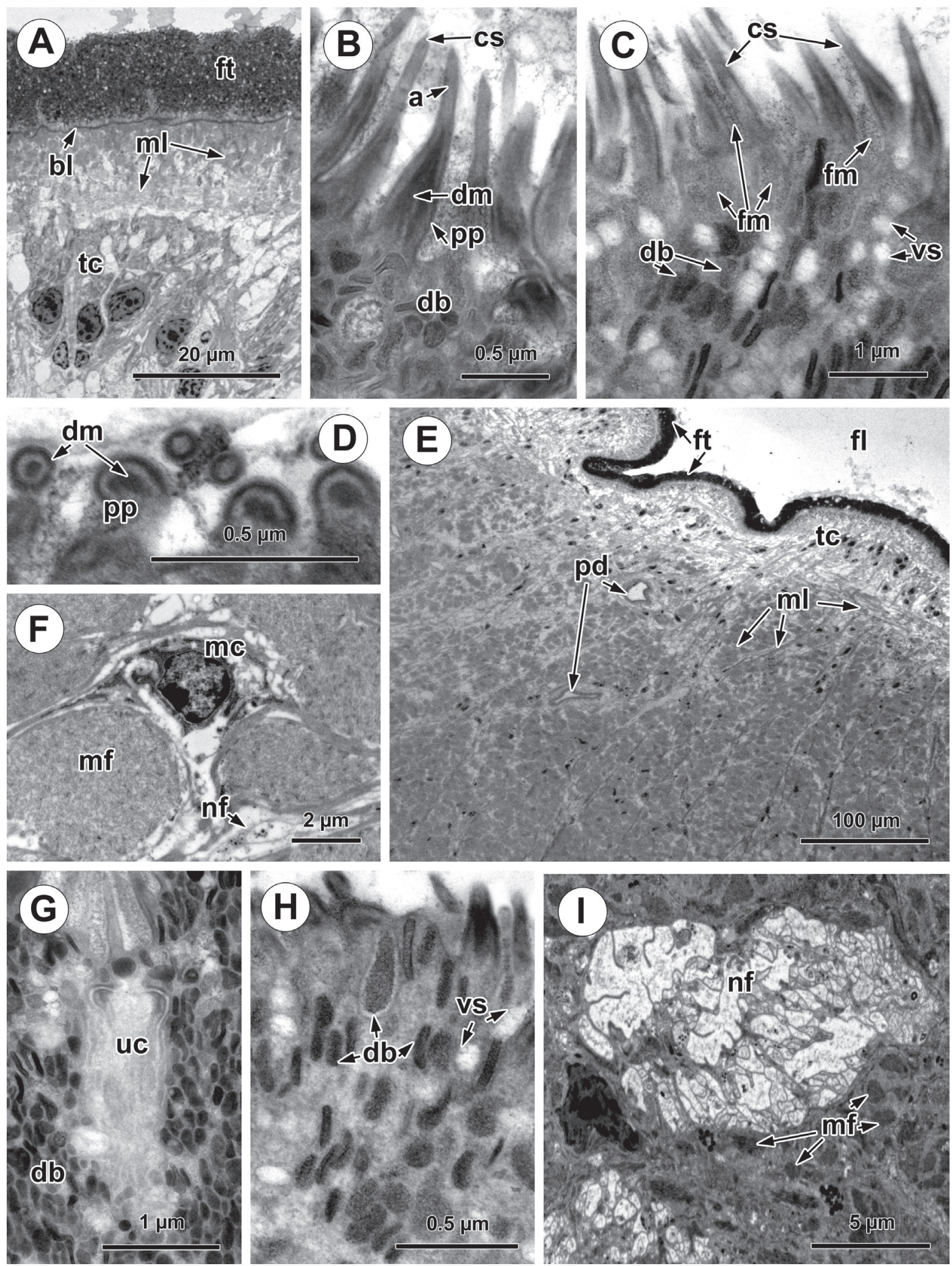

Fig. 4. Transmission electron micrographs of views of the funnel-shaped canal of mature Gyrocotyle urna Wagener, 1855 from Chimaera monstrosa Linnaeus. A - syncytial cytoplasm with sunken perikarya of the funnel-shaped canal; B - conical surface structures showing their expanded proximal part with aggregation of dense material and narrow apical part; $\mathbf{C}-$ inclusions of the syncytial cytoplasm; D - cross-section of conical surface structures; $\mathbf{E}$ - section through the funnel-shaped canal showing differently directed muscle layers; $\mathbf{F}$ - muscle cell between muscle fibres; $\mathbf{G}$ - uniciliated receptor within tegumental syncytial cytoplasm; $\mathbf{H}$ - tegumental inclusions; I - portion of the posterior nerve cord between muscle fibrils. Abbreviations: a - apical part of conical surface structure; bl - basal lamina of tegumental syncytial cytoplasm; cs - conical surface structures of the funnel-shaped canal tegument; db - dense bodies; $\mathrm{dm}$ - aggregation of dense material into conical surface structures; $\mathrm{fl}$ - funnel-shaped canal lumen; fm - flocculent material of dense bodies; $\mathrm{ft}$ - funnel-shaped canal tegument; $\mathrm{mc}$ - muscle cell; $\mathrm{mf}$ - muscle fibrils; $\mathrm{ml}$ - muscle layers; $\mathrm{nf}$ - nerve fibres; $\mathrm{pd}$ - protonephridial duct; pp - proximal part of conical surface structures; tc - tegumental cells; uc - uniciliate receptor; vs - vesicle. 
The funnel-shaped canal is narrow, about 2.5-3.0 mm long (Fig. 3B,C,E,G) and turns abruptly dorsally at its end to open on the dorsal surface on the median line (Fig. 3D). The aperture of this canal has a diameter of about $300 \mu \mathrm{m}$ and opens at a rounded elevation that is about $900 \mu \mathrm{m}$ in diameter (Fig. 3D, inset).

The tegumental syncytial cytoplasm of the funnelshaped canal is about $11-14 \mu \mathrm{m}$ in thickness (Fig. 4A) and is covered with conical surface structures up to $1.5 \mu \mathrm{m}$ long (Figs. 3H, 4B,C). They have an expanded proximal portion about $0.7 \mu \mathrm{m}$ long and $0.3 \mu \mathrm{m}$ in diameter at their widest portion (Figs. 3H, 4B). Along the length of the proximal portion an aggregation of electron-dense material is present (Fig. 4B,C). In cross-section, this material is concentrated as a ring immediately under the plasma membrane that is covered by the conical surface structures; the electron-dense material also occupies the central area of the proximal portion (Fig. 4D). The thin apical part of these structures is about $0.5 \mu \mathrm{m}$ long and $0.08 \mu \mathrm{m}$ wide and has a homogeneous, moderately dense content (Fig. 4B,D). The syncytial cytoplasm of the tegument is filled with electron-dense bodies and moderately dense or electron-lucent vesicles (Fig. 4B,C,H). Electron-dense bodies contain dark flocculent material (Fig. 4H). Frequently, the plasma membrane of these bodies is in contact with the surface membrane of the tegument (Fig. 4B,C), and the flocculent content can be observed between surface structures (Fig. 4B,C). Sunken perikarya are grouped under the syncytial cytoplasm (Fig. 4A). Nerve endings are distributed in the tegumental cytoplasm (Fig. 4G). The space under the funnel tegument is occupied by differently directed muscle layers (Figs. 3B,E,F, 4E). Numerous myocytons, nerve fibres and protonephridial ducts are seen between the muscle fibrils (Fig. 4F,E,I). The nerve cord is present at the base of the funnel (Fig. 4I).

\section{DISCUSSION}

\section{Anterior organ of the Gyrocotylidea}

The present study on the anterior organ and posterior funnel-shaped canal of the gyrocotylidean Gyrocotyle urna has shown their ultrastructural characteristics for the first time. TEM examination reveals that the lumen of the anterior organ of G. urna is a closed prolongation of the body tegument formed by tegumental syncytial cytoplasm with sunken perikarya. The tegumental syncytial cytoplasm of the proper anterior organ is slightly thinner than that of the body tegument and includes a large number of vacuoles together with electron-dense bodies and vesicles (Lyons 1969, Poddubnaya et al. 2006). The luminal plasmalemma of the anterior organ, as the body tegument, bears filitriches, but they are shorter and of irregular length. Bandoni and Brooks (1987) compared the anterior end of species of Gyrocotyle with the mouth of monogeneans that is rarely encircled by an oral sucker unlike that of digeneans (Fried and Haseeb 1991). The anterior organ (or acetabulum, or anterior pit) of species of Gyrocotyle looks like the mouth of monogeneans or an attachment sucker-like organ of some evolutionary early divergent tapeworms that possess a single apical anterior sucker-like organ (Riggenbach 1899, Fried and Haseeb 1991, Olson and Caira 2001, Kuchta et al. 2014).

TEM examinations of the digestive system of the Monogenea have shown that the proper mouth cavity of the monogeneans is lined by the tegument that is identical and continuous with the surface tegument, whereas the subsequent anterior digestive tract of monopisthocotylean monogeneans is a complex structure with muscular, glandular and sensory components and epithelial modifications of its different parts (Halton and Stranock 1976, Junchis 1990). The anterior 'buccal complex' of most polyopisthocotylean monogeneans studied (Halton and Morris 1975, Rohde 1979, Rohde and Watson 1996, Valigurová et al. 2011) is a complex structure with numerous glands that are associated with their foregut (Fried and Haseeb 1991, Rohde and Watson 1996). It should be noted that in gyrocotylideans, this apical anterior invagination is not associated with the digestive system that is absent in all cestodes, but it represents a muscular invagination thought to be a vestigial pharynx in all monogeneans (Brooks et al. 1985).

Interestingly, an apical attachment sucker-like organ is also present in species of some cestode orders, such as the spathebothriidean Cyathocephalus truncatus (Pallas, 1781) from freshwater teleosts, the diphyllobothriidean Scyphocephalus bisulcatus Riggenbach, 1899 from reptiles, and, species of the order Lithobothriidea, parasites of elasmobranchs (Riggenbach 1899, Olson and Caira 2001, Kuchta et al. 2014)

According to Watson (1911) and Williams et al. (1987), the anterior organ of gyrocotylideans has never been found attached or functioning as a true sucker, but these authors observed the movements of this organ in live specimens. The present study supports, to some extent, the abovementioned observations because a well-developed muscular complex penetrated by numerous nerve fibres and receptors was observed around the pore of the anterior organ. It is interesting to note that Terenina et al. (2009) revealed the same pattern of the arrangement of muscle fibres with nerve fibres surrounding an apical invagination of the scolex of the spathebothriidean C. truncatus.

\section{Funnel-shaped canal of the Gyrocotylidea}

The present ultrastructural study has revealed that the funnel-shaped canal is a specialised, muscular part of the posterior attachment organ of the Gyrocotylidea, the tegumental layer of which (i) is covered with unique conical sclerite-like surface structures, (ii) produces electron-dense bodies, the flocculent material of which is transported into the canal lumen, and (iii) is surrounded by a well-developed thick muscle area mixed with numerous nerve fibres. It should be noted that the transport of cytoplasmic bodies of the tegument to the surface plasma membrane has been reported for many platyhelminths and their production is an active process induced by specific physiological and biochemical triggers in individual body areas (Pappas and Read 1975). 
Concerning the development of the funnel-shaped canal in the Gyrocotylidea, an investigation of early stages of the development of the postlarvae of G. urna and G. fimbriata Watson, 1911 by Lynch (1945) has shown that their rosette has the form of a circular flange growing out from around the body just anterior to the hook-bearing posterior appendage called the cercomer of the larva (decacanth). The border of the rosette remains circular until the development of plications begins. The dorsal pore appears just anterior to the base of the cercomer. Halvorsen and Williams (1968) indicated that postlarvae of G. urna attach to the apex of a villus of the spiral valve of chimaera by means of ten hooks on the cercomer. The tissues immediately in front of the larval cercomer then grow and extend over the apex of the host villus to form a prominent, inverted cup-shaped organ of attachment. When this structure develops and its cavity deepens, the larval cercomer is pushed deep into the cup.

According to Bychowsky (1957), the development of a haptor in polyopisthocotylean monogeneans is similar to that of the funnel-shape canal in gyrocotylideans. In contrast, Junchis (1990) and Rohde and Watson (1995) revealed that the posterior attachment organ is an extension of the body proper and its tegument is continuous with that of the main body and covered with the same surface structures like their body tegument in monopisthocotylean monogeneans such as species of Ancyrocephalus Creplin, 1839, Anoplodiscus Sonsino, 1890, Dactylogyrus Diesing, 1850 and Udonella Johnston, 1835. Our present and previous investigations (Poddubnaya et al. 2008) have shown the presence of specialised types of surface structures on the funnel-shaped organ of G. urna: electron-dense conical sclerite-like structures (luminal surface funnel-shaped canal), dense lanceolate structures (inner surface of the rosette folds) and digitiform structures (outer surface of the rosette folds). This fact supports Bychowsky's (1957) opinion that the posterior attachment organ of gyrocotylideans is not an extension of the body proper as it is in monopisthocotylean monogeneans.

Watson (1911), Halvorsen and Williams (1968) and Williams et al. (1987) claimed that the posterior attachment organ of gyrocotylideans is powerful. They observed that the series of waves of contraction passes from the margin of the rosette towards the canal opening during the attachment of the rosette and that the funnel opening is closed as long as the parasite remains attached, enabling the worms to move to another area of the spiral valve of its host. Mucus is accumulated within the funnel when the rosette is attached to the gut (Halvorsen and Williams 1968). In addition, three different types of specialised secretory glands with secretions comprising different types of granules that are discharged on the inner surface of the rosette of the gyrocotylideans have been described (Poddubnaya et al. 2008). Based on the similarity between secretory glands of the posterior rosette of Gyrocotyle and those in the anterior attachment glands of monogeneans, it is possible to assume that the glands of the Gyrocotylidea may aid in temporary attachment to the host tissue (Poddubnaya et al. 2008).

\section{Phylogenetic remarks}

Llewellyn (1987) speculated that "an ancient monogenean divergence was a stock which entered the gut of holocephalans, lost its own gut, reduced the complement of larval marginal hooks from 16 to 10 and developed a powerful posterior pseudohaptor ('rosette organ')." Cartilaginous chimaeras represent the oldest extant group of fish (Inoue et al. 2010) and have evolved a quite unique parasite fauna, most members of which co-evolved with their host, such as monogeneans of the family Chimaericolidae Brinkmam, 1942 (Polyopisthocotylea) and cestodes of the order Gyrocotylidea (see Bandoni and Brooks 1987, Williams et al. 1987, Lebedev 1995).

The ultrastructural investigations on G. urna have highlighted some ultrastructural characters that may be useful for unravelling the evolutionary history of the Gyrocotylidea and their phylogenetic relations with other parasitic flatworms (Neodermata). These characters of potential phylogenetic importance include: (i) capilliform filitriches on the body surface of gyrocotylideans and 'true' tapeworms (Eucestoda); (ii) laminate nature of gyrocotylidean neodermal sclerites that somewhat resembe calcareous corpuscles of eucestodes; (iii) similarity between secretory glands of the posterior rosette of G. urna and those in the anterior attachment glands of monogeneans; (iv) the structural arrangement of the protonephridial terminal organ in gyrocotylideans and some monogeneans; (v) apical structures of protonephridial canal walls of gyrocotylideans and spathebothriidean tapeworms; (vi) cellular nature of protonephridial ducts in gyrocotylideans, monogeneans and trematodes; (vii) the similarity in the degree of division of the ovary and arrangement and structure of associated ducts in G. urna and the early derived monogenean genus Chimaericola Brinkmann, 1942; (viii) discriminatory characters of the cytoarchitecture of the vitelline follicles of gyrocotylideans and eucestodes; (ix) unique uterine ultrastructural pattern with the cellular nature of the uterine tube of gyrocotylideans, monogeneans and trematodes; (x) uterine glands of the middle portion of the uterine tube in gyrocotylideans and spathebothriideans; and (xi) spermatozoon with mitochondria in gyrocotylideans, monogeneans and trematodes (Xylander 2001, Poddubnaya et al. 2006, 2008, 2009, 2010, 2012, Xylander and Poddubnaya 2009).

The present study has shown that the anterior suckerlike organ of the Gyrocotylidea is a closed muscular invagination of the body tegument with an apical pore and somewhat resembles the anterior attachment organ of the spathebothriidean C. truncatus and the diphyllobothriidean S. bisulcatus, which belongs among the earliest derived branched of 'true' tapeworms (Eucestoda) (Waechenbach et al. 2012, Kuchta et al. 2014). If the gyrocotylidean anterior sucker-like organ is an indication of the previous presence of a digestive system in ancient ancestors of the Gyrocotylidea, such an organ may have been modified into a sucker-like attachment organ of the Eucestoda during cestode evolution. In fact, gyrocotylideans share some ultrastructural characters with spathebothriideans, such as 
the luminal surface structure of protonephridial ducts and uterine glands of the middle portion of the uterine tube. In contrast, the unique posterior funnel-shaped attachment organ of the Gyrocotylidea resembles the haptor of polyopisthocotylean monogeneans in its position at the posterior end of the body and presumed origin (Lynch 1945, Bychowsky 1957, Halvorsen and Williams 1968, Williams et al. 1987). The above-mentioned features tend to support the basal position of the Gyrocotylidea among cestodes. In addition, they also indicate a possible close relationship of gyrocotylidean ancestors with monogeneans.

Acknowledgements. The present study was supported by the Russian Foundation for Fundamental Research (projects Nos. 12-04-00149 and 15-04-02890 to L.P.G.) and partly by the Institute of Parasitology (RVO: 60077344) and the Czech Science Foundation (project No. P505/12/G112). The authors would like to thank the staff of the Centre of Electron Miscroscopy, I.D. Papanin Institute for Biology of Inland Waters, Russian Academy of Sciences, Russia, for the technical assistence.

\section{REFERENCES}

BAndoni S.M., Brooks D.R. 1987: Revision and phylogenetic analysis of the Gyrocotylidea Poche, 1926 (Platyhelminthes: Cercomeria: Cercomeromorpha). Can. J. Zool. 65: 2369-2389.

Bristow G.A. 1992: On the distribution, ecology and evolution of Gyrocotyle urna, G. confusa and G. nybelini (Cercomeromorpha: Gyrocotylidea) and their host Chimaera monstrosa (Holocephalida; Chimaeridae) in Norwegian waters, with a review of the species question. Sarsia 77: 119-124.

Brooks D.R., O'Grady R.T., Glen D.R. 1985: The phylogeny of the Cercomeria Brooks, 1982 (Platyhelminthes). Proc. Helminthol. Soc. Wash. 52: 1-20.

Bychowsky B.E. 1957. [The Monogenea, Their Systematics and Phylogeny.] AN SSSR, Moscow - Leningrad, 509 pp. (In Russian.)

Chervy L. 2009: Unified terminology for cestode microtriches: a proposal from the International Workshops on Cestode Systematics in 2001-2008. Folia Parasitol. 56: 199-230.

Fried B., Haseeb M.F. 1991: Platyhelminthes: Aspidogastera, Monogenea and Digenea. In: F.W. Harrison and B.J. Bogitsh (Eds.), Microscopic Anatomy of Invertebrates. Volume 3: Platyhelminthes and Nemertinea, Chapter 3. Willey-Liss, Inc., New York, pp. 141-219.

Gibson D.I. 1994: Gyrocotylidea Poche, 1926. In: L.F. Khalil, A. Jones and R.A. Bray (Eds.), Keys to the Cestode Parasites of Vertebrates. CAB International, Wallingford, pp. 11-13.

Halton D.W., Morris G.P. 1975: Ultrastructure of the anterior alimentary tract of a monogenean, Diclidophora merlangi. Int. J. Parasitol. 5: 407-419.

Halton D.W., STRANOCK S.D. 1976: Ultrastructure of the foregut and associated glands of Calicotyle kroyeri (Monogenea: Monopisthocotylidea). Int. J. Parasitol. 6: 517-526.

Halvorsen O., Williams H.H. 1968: Studies of the helminth fauna of Norway. IX. Gyrocotyle (Platyhelminthes) in Chimaera monstrosa from Oslo fjord, with emphasis on its mode of attachment and a regulation in the degree of infection. Nytt Mag. Zool. 15: $130-142$

Inoue J.C., Miya M., Lam K., Tay B.H., Danks J.A., Bell J., WALKeR T.I., VenkAtesh B. 2010: Evolutionary origin and phylogeny of the modern holocephalans (Chondrichthyes: Chimaeriformes): a mitogenomic perspective. Mol. Biol. Evol. 27: 2576-2586.

JUNCHIS L.A. 1990: [Fine structure of the anterior alimentary tract of some species of lower monogeneans.] Proc. Zool. Inst., Academy of Science of the USSR, 221: 17-25. (In Russian.)

Kuchta R., Pearson R., Scholz T., Ditrich O., Olson P.D. 2014: Spathebothriidea: survey of species, scolex and egg morphology, and interrelationships of a non-segmented, relictual tapeworm group (Platyhelminthes: Cestoda). Folia Parasitol. 61: 331-346.

LeBedev B.I. 1995: [Essays on Biodiversity and Evolutionary Parasitology.] Dalnauka, Vladivostok, 208 pp. (In Russian.)

Llewellyn J. 1987: Phylogenetic inference from platyhelminth life-cycle stages. Int. J. Parasitol. 17: 281-289.
Lockyer A.E., Olson P.D., Littlewood D.T.J. 2003: Utility of complete large and small subunit rRNA genes in resolving the phylogeny of the Neodermata (Platyhelminthes): implications and a review of the cercomer theory. Biol. J. Linn. Soc. 78: $155-171$.

LYNCH J.E. 1945: Redescription of the species of Gyrocotyle from the ratfish, Hydrolagus colliei (Lay and Bennet), with notes on the morphology and taxonomy of the genus. J. Parasitol. 31: 418-446.

Lyons K.M. 1969: The fine structure of the body wall of Gyrocotyle urna. Z. Parasitenkd. 33: 95-109.

Olson P.D., CaIRa J.N. 2001: Two new species of Litobothrium Dailey, 1969 (Cestoda: Litobothriidea) from thresher sharks in the Gulf of California, Mexico, with redescriptions of two species in the genus. Syst. Parasitol. 48: 159-177.

Olson P.D., Littlewood D.T.J., Bray R.A., Mariaux J. 2001: Interrelationships and evolution of the tapeworms (Platyhelminthes: Cestoda). Mol. Phyl. Evol. 19: 443-467.

PAPPAS P.W., READ C.P. 1975: Membrane transport in helminth parasites: a review. Exp. Parasitol. 37: 469-530.

Poddubnaya L.G., Bruňanská M., Kuchta R., Scholz T. 2006: First evidence of the presence of microtriches in the Gyrocotylidea. J. Parasitol. 92: 703-707.

Poddubnaya L.G., Kuchta R., Levron C., Gibson D.I., Scholz T. 2009: The unique ultrastructure of the uterus of the Gyrocotylidea Poche, 1926 (Cestoda) and its phylogenetic implications. Syst. Parasitol. 74: 81-93.

Poddubnaya L.G., Kuchta R., Scholz T., Xylander W.E.R. 2010: Ultrastructure of the ovarian follicles, oviducts and oocytes of Gyrocotyle urna (Neodermata: Gyrocotylidea). Folia Parasitol. 57: 173-184.

Poddubnaya L.G., Scholz T., Kuchta R., Levron C., Gibson D.I. 2008: Ultrastructure of the surface structures and secretory glands of the rosette attachment organ of Gyrocotyle urna (Cestoda: Gyrocotylidea). Folia Parasitol. 55: 207-218.

Poddubnaya L.G., Xylander W.R.R., Gibson D.I. 2012: U1trastructural characteristics of the protonephridial terminal organ and associated ducts of adult specimens of the Aspidogastrea, Digenea and Monogenea, with comments on the relationships between these groups. Syst. Parasitol. 82: 89-104.

Riggenbach E. 1899. Scyphocephalus bisulcatus n. g. n. sp, ein neuer Reptiliencestode. Zool. Jahrb., Syst., Geogr. Biol. Thiere 12: 145-153.

Rohde K. 1979: The buccal organ of some Monogenea Polyopisthocotylea. Zool. Scr. 8: 161-170.

RoHDE K., WATSON N.A. 1995: Comparative ultrastructural study of the posterior suckers of four species of symbiotic Platyhelminthes, Temnocephala sp. (Temnocephalida), Udonella caligorum (Udonellidea), Anoplodiscus cirrusspiralis (Monogenea: Monopisthocotylea), and Philophthalmus sp. (Trematoda: Digenea). Folia Parasitol. 42: 11-28.

Rohde K., WAtson N.A. 1996: Ultrastructure of the buccal complex of Pricea multae (Monogenea: Polyopisthocotylea, Gastrocotylidae). Folia Parasitol. 43: 117-132. 
Terenina N.B., Poddubnaya L.G., Tolstenkov O.O., GusTAFSSON M.K.S. 2009: An immunocytochemical, histochemical and ultrastructural study of the nervous system of the tapeworm Cyathocephalus truncatus (Cestoda, Spathebothriidea). Parasitol. Res. 104: 267-275.

Valigurová A., Hodová I., Sonnek R., Koubková B., Gelnar M. 2011: Eudiplozoon nipponicum in focus: monogenean exhibiting a highly specialized adaptation for ectoparasitic life style. Parasitol. Res. 108: 383-394.

Waeschenbach A., Webster B.L., Bray R.A., Littlewood D.T.J. 2007: Added resolution among ordinal level relationship of tapeworms (Platyhelminthes: Cestoda) with complete small and large subunit nuclear ribosomal RNA genes. Mol. Phyl. Evol. 45: 311-325.

Waeschenbach A., Webster B.L., Littlewood D.T.J. 2012: Adding resolution to ordinal level relationships of tapeworms
(Platyhelminthes: Cestoda) with large fragments of mtDNA. Mol. Phyl. Evol. 63: 834-847.

Watson E.E. 1911: The genus Gyrocotyle, and its significance for problems of cestode structure and phylogeny. Univ. Calif. Publ. Zool. 6: 353-468.

Williams H.H., Colin J.A., Halvorsen O. 1987: Biology of gyrocotylideans with emphasis on reproduction, population ecology and phylogeny. Parasitology 95: 173-207.

XYlander W.E.R. 2001: The Gyrocotylidea, Amphilinidea and the early evolution of Cestoda. In: D.T.J. Littlewood and R.A. Bray (Eds.), Interrelationships of the Platyhelminthes. Taylor and Francis, London, pp. 103-111.

Xylander W.E.R., Poddubnaya L.G. 2009: Ultrastructure of the neodermal sclerites of Gyrocotyle urna Grube and Wagener, 1852 (Gyrocotylidea, Cestoda). Parasitol. Res. 105: 1593-1601.

Cite this article as: Poddubnaya L.G., Kuchta R., Bristow G.A., Scholz T. 2015: Ultrastructure of the anterior organ and posterior funnel-shaped canal of Gyrocotyle urna Wagener, 1852 (Cestoda: Gyrocotylidea). Folia Parasitol. 62: 027. 